\title{
Analysis of an Impulsive Predator-Prey System with Monod-Haldane Functional Response and Seasonal Effects
}

\author{
Hunki Baek, ${ }^{1}$ Younghae Do, ${ }^{1}$ and Yasuhisa Saito ${ }^{2}$ \\ ${ }^{1}$ Department of Mathematics, Kyungpook National University, Daegu 702-701, South Korea \\ ${ }^{2}$ Department of Mathematics, Pusan National University, Pusan 609-735, South Korea \\ Correspondence should be addressed to Hunki Baek, hkbaek@knu.ac.kr
}

Received 5 December 2008; Revised 23 March 2009; Accepted 27 April 2009

Recommended by J. Rodellar

For a class of impulsive predator-prey systems with Monod-Haldane functional response and seasonal effects, we investigate conditions for the local and global stabilities of prey-free solutions and for the permanence of the system by using the Flquet theory of impulsive differential equations and comparison techniques. In addition, we numerically analyze the phenomena caused by seasonal effects and impulsive perturbation. It will be applicable to the controllability for the population of prey and predator.

Copyright (C) 2009 Hunki Baek et al. This is an open access article distributed under the Creative Commons Attribution License, which permits unrestricted use, distribution, and reproduction in any medium, provided the original work is properly cited.

\section{Introduction}

One of main goals in population dynamics is to understand the dynamical relationship between predator and prey. Such relationship can be represented as the functional response which refers to the change in the density of prey attached per unit time per predator as the prey density changes. Holling [1] gave three different kinds of functional response for different kinds of species to model the phenomena of predation. These functional responses are monotonic in the first quadrant. But, some experiments and observations indicate that nonmonotonic response occurs at a level [2]. To model such an inhibitory effect, Andrews [2] had suggested a function

$$
p(x)=\frac{m x}{a+b x+x^{2}}
$$

called the Monod-Haldane function. Sokol and Howell [3] had proposed a simplified MonodHaldane function having the form

$$
p(x)=\frac{m x}{a+x^{2}}
$$


Thus, the Monod-Haldane type predator-prey model can be described by the following differential equation $[1,4-7]$ :

$$
\begin{gathered}
x^{\prime}(t)=r x(t)\left(1-\frac{x(t)}{K}\right)-\frac{x(t) y(t)}{1+e x^{2}(t)} \\
y^{\prime}(t)=-d y(t)+\frac{c x(t) y(t)}{1+e x^{2}(t)}
\end{gathered}
$$

where $x(t)$ and $y(t)$ represent population densities of prey and predator at time $t$. All parameters are positive constants. Usually, $r$ is the intrinsic growth rate of the prey, $K$ is the carrying capacity of the prey, the constant $d$ is the death rate of the predator, $c$ is the rate of conversion of a consumed prey to a predator and $e$ measures the level of prey interference with predation.

As well known, there are a number of factors in the environment, which vary with changing seasons and affect various parameters in the ecological models. In fact, countless organisms live in seasonally or diurnally forced environments. For the reason, it is necessary and important to consider models with periodic ecological parameters. There are several ways to apply periodic perturbation in an ecological model [8-10]. In this paper we consider the intrinsic growth rate $r$ in the model (1.3) as periodically varying function of time due to seasonal variation. The seasonality is superimposed as follows:

$$
r_{0}=r(1+\epsilon \sin (\omega t)),
$$

where the parameter $\epsilon$ represents the degree of seasonality, $\lambda=r \epsilon$ is the magnitude of the perturbation in $r_{0}$, and $\omega$ is the angular frequency of the fluctuation caused by seasonality.

Moreover, there are still some other perturbations such as fire, flood, harvesting seasons, and so forth, that are not suitable to be considered continually. These impulsive perturbations bring sudden change to the model. Thus, it is natural to assume that these perturbations act instantaneously, that is, in the form of impulse [11-16].

In this paper, with the ideas discussed above, we consider the following predator-prey system with periodic constant impulsive immigration of the predator and periodic variation in the intrinsic growth rate of the prey:

$$
\begin{gathered}
x^{\prime}(t)=r x(t)\left(1-\frac{x(t)}{K}\right)-\frac{x(t) y(t)}{1+e x^{2}(t)}+\lambda x(t) \sin (\omega t), \\
y^{\prime}(t)=-d y(t)+\frac{c x(t) y(t)}{1+e x^{2}(t)}, \quad t \neq n \tau, \\
\Delta x(t)=0, \\
\Delta y(t)=p, \quad t=n \tau, \\
\left(x\left(0^{+}\right), y\left(0^{+}\right)\right)=\left(x_{0}, y_{0}\right),
\end{gathered}
$$

where $\Delta z(t)=z\left(t^{+}\right)-z(t), \tau$ is the period of the impulsive immigration or stock of the predator, $p$ is the size of immigration or stock of the predator. The parameters $\lambda$ and $\omega$ 
represent the amplitude and frequency of the forcing term, respectively. When $\lambda=0$ and $p=0$, system (1.5) coincides with (1.3).

Impulsive control methods can be found in almost every field of applied sciences. The theoretical investigation and its application can be found in Bainov and Simeonov [17] and Lakshmikantham et al. [18]. Furthermore, the impulsive differential equations dealing with population dynamics are literate in [9, 13, 14, 16, 19-23]. Especially, Zhang et al. [22] studied system (1.5). They investigated the abundance of complex dynamics of system (1.5) and suggested a more executable way for observing chaos and coexistence of attractors by using numerical simulations. Thus, the purpose of this paper is to investigate dynamical behaviors of system (1.5) theoretically. In this context, we find conditions for the local and global stabilities of pest-free periodic solutions and for the permanence of system (1.5) by using the Floquet theory and comparison techniques. Moreover, numerical simulations for the effects of periodic forcing and impulsive perturbations are illustrated.

\section{Notations and Lemmas}

In this section, we give some notations, definitions and lemmas which will be useful for our main results.

Let $\mathbb{R}_{+}=[0, \infty), \mathbb{R}_{+}^{*}=(0, \infty)$, and $\mathbb{R}_{+}^{2}=\left\{\mathbf{x}=(x, y) \in \mathbb{R}^{2}: x, y \geq 0\right\}$. Denote $\mathbb{N}$ the set of all of nonnegative integers and $F=\left(F_{1}, F_{2}\right)^{T}$ the right hand side of the model (1.5). Let $V: \mathbb{R}_{+} \times \mathbb{R}_{+}^{2} \rightarrow \mathbb{R}_{+}$, then $V$ is said to be in a class $V_{0}$ if

(1) $V$ is continuous on $((n-1) \tau, n \tau] \times \mathbb{R}_{+}^{2}$, and $\lim _{(t, \mathbf{y}) \rightarrow(n \tau, \mathbf{x}), t>n \tau} V(t, \mathbf{y})=V\left(n \tau^{+}, \mathbf{x}\right)$ exists.

(2) $V$ is locally Lipschitzian in $\mathbf{x}$.

Definition 2.1. Let $V \in V_{0},(t, \mathbf{x}) \in((n-1) \tau, n \tau] \times \mathbb{R}_{+}^{2}$. The upper right derivatives of $V(t, \mathbf{x})$ with respect to the impulsive differential system (1.5) is defined as

$$
D^{+} V(t, \mathbf{x})=\limsup _{h \rightarrow 0+} \frac{1}{h}[V(t+h, \mathbf{x}+h f(t, \mathbf{x}))-V(t, \mathbf{x})]
$$

Remark 2.2. (1) The solution of system (1.5) is a piecewise continuous function $\mathbf{x}: \mathbb{R}_{+} \rightarrow \mathbb{R}_{+}^{2}$. That is, $\mathbf{x}(t)$ is continuous on $((n-1) \tau, n \tau], n \in \mathbb{N}$ and $\mathbf{x}\left(n \tau^{+}\right)=\lim _{t \rightarrow n \tau^{+}} \mathbf{x}(t)$ exists.

(2) The smoothness properties of $f$ guarantees the global existence and uniqueness of solution of system (1.5); see [18] for the details.

We will use the following important comparison theorem on an impulsive differential equation [18].

Lemma 2.3 (Comparison theorem). Suppose $V \in V_{0}$ and

$$
\begin{array}{ll}
D^{+} V(t, \mathbf{x}) \leq g(t, V(t, \mathbf{x})), & t \neq n \tau, \\
V\left(t, \mathbf{x}\left(t^{+}\right)\right) \leq \psi_{n}(V(t, \mathbf{x})), & t=n \tau
\end{array}
$$


$g: \mathbb{R}_{+} \times \mathbb{R}_{+} \rightarrow \mathbb{R}$ is continuous on $((n-1) \tau, n \tau] \times \mathbb{R}_{+}$and for $u(t) \in \mathbb{R}_{+}, n \in \mathbb{N}$, $\lim _{(t, y) \rightarrow\left(n \tau^{+}, u\right)} g(t, y)=g\left(n \tau^{+}, u\right)$ exists, $\psi_{n}: \mathbb{R}_{+} \rightarrow \mathbb{R}_{+}$is nondecreasing. Let $r(t)$ be the maximal solution of the scalar impulsive differential equation

$$
\begin{gathered}
u^{\prime}(t)=g(t, u(t)), \quad t \neq n \tau, \\
u\left(t^{+}\right)=\psi_{n}(u(t)), \quad t=n \tau, \\
u\left(0^{+}\right)=u_{0},
\end{gathered}
$$

existing on $[0, \infty)$. Then $V\left(0^{+}, \mathbf{x}_{0}\right) \leq u_{0}$ implies that $V(t, \mathbf{x}(t)) \leq r(t), t \geq 0$, where $\mathbf{x}(t)$ is any solution of (2.2).

We now indicate a special case of Lemma 2.3 which provides estimations for the solution of a system of differential inequalities. For this, we let $P C\left(\mathbb{R}_{+}, \mathbb{R}\right)\left(P C^{1}\left(\mathbb{R}_{+}, \mathbb{R}\right)\right)$ denote the class of real piecewise continuous (real piecewise continuously differentiable) functions defined on $\mathbb{R}_{+}$.

Lemma 2.4 (see [18]). Let the function $u(t) \in P C^{1}\left(\mathbb{R}^{+}, \mathbb{R}\right)$ satisfy the inequalities

$$
\begin{gathered}
u^{\prime}(t) \leq f(t) u(t)+h(t), \quad t \neq \tau_{k}, \quad t>0, \\
u\left(\tau_{k}^{+}\right) \leq \alpha_{k} u\left(\tau_{k}\right)+\theta_{k}, \quad k \geq 0 \\
u\left(0^{+}\right) \leq u_{0},
\end{gathered}
$$

where $f, h \in P C\left(\mathbb{R}_{+}, \mathbb{R}\right)$ and $\alpha_{k} \geq 0, \theta_{k}$ and $u_{0}$ are constants, and $\left(\tau_{k}\right)_{k \geq 0}$ is a strictly increasing sequence of positive real numbers. Then, for $t>0$,

$$
\begin{aligned}
u(t) \leq & u_{0}\left(\prod_{0<\tau_{k}<t} \alpha_{k}\right) \exp \left(\int_{0}^{t} f(s) d s\right)+\int_{0}^{t}\left(\prod_{s \leq \tau_{k}<t} \alpha_{k}\right) \exp \left(\int_{s}^{t} f(\gamma) d \gamma\right) h(s) d s \\
& +\sum_{0<\tau_{k}<t}\left(\prod_{\tau_{k}<\tau_{j}<t} \alpha_{j}\right) \exp \left(\int_{\tau_{k}}^{t} f(\gamma) d \gamma\right) \theta_{k} .
\end{aligned}
$$

Similar result can be obtained when all conditions of the inequalities in Lemmas 2.3 and 2.4 are reversed. Using Lemma 2.4, it is possible to prove that the solutions of the Cauchy problem (2.3) with strictly positive initial value remain strictly positive.

Lemma 2.5. The positive octant $\left(\mathbb{R}_{+}^{*}\right)^{2}$ is an invariant region for system (1.5). 
Proof. Let $(x(t), y(t)):\left[0, t_{0}\right) \rightarrow \mathbb{R}^{2}$ be a solution of system (1.5) with a strictly positive initial value $\left(x_{0}, y_{0}\right)$. By Lemma 2.4 , we can obtain that, for $0 \leq t<t_{0}$,

$$
\begin{aligned}
& x(t) \geq x_{0} \exp \left(\int_{0}^{t} g_{1}(s) d s\right), \\
& y(t) \geq y_{0} \exp \left(\int_{0}^{t} g_{2}(s) d s\right),
\end{aligned}
$$

where $g_{1}(s)=r(1-x(s) / K-y(s))$ and $g_{2}(s)=-d$. Thus, $x(t)$ and $y(t)$ remain strictly positive on $\left[0, t_{0}\right)$.

Now, we give the basic properties of the following impulsive differential equation

$$
\begin{gathered}
y^{\prime}(t)=-d y(t), \quad t \neq n \tau, \\
y\left(t^{+}\right)=y(t)+p, \quad t=n \tau, \\
y\left(0^{+}\right)=y_{0} .
\end{gathered}
$$

Then we can easily obtain the following results.

Lemma 2.6. (1) $y^{*}(t)=p \exp (-d(t-(n-1) \tau)) /(1-\exp (-d \tau)), t \in((n-1) \tau, n \tau], n \in \mathbb{N}$ and $y^{*}\left(0^{+}\right)=p /(1-\exp (-d \tau))$ is a positive periodic solution of $(2.7)$.

(2) $y(t)=\left(y\left(0^{+}\right)-p /(1-\exp (-d \tau))\right) \exp (-d t)+y^{*}(t)$ is the solution of (2.7) with $y_{0} \geq 0$, $t \in((n-1) \tau, n \tau]$ and $n \in \mathbb{N}$. $t \rightarrow \infty$.

(3) All nonnegative solutions $y(t)$ of (2.7) tend to $y^{*}(t)$. That is, $\left|y(t)-y^{*}(t)\right| \rightarrow 0$ as

It is from Lemma 2.6(3) that the general solution $y(t)$ of (2.7) can be synchronized with the positive periodic solution $y^{*}(t)$ of (2.7) for sufficiently large $t$ and we can obtain the complete expression for the prey-free periodic solution of system (1.5):

$$
\left(0, y^{*}(t)\right)=\left(0, \frac{p \exp (-d(t-n \tau))}{1-\exp (-d \tau)}\right) \text { for } t \in((n-1) \tau, n \tau]
$$

The boundness of the solutions of system (1.5) was proven by [22] as follows.

Theorem 2.7 (see [22]). There is an $R>0$ such that $x(t), y(t) \leq R$ for all $t$ large enough, where $(x(t), y(t))$ is a solution of the model (1.5).

\section{Main Theorems}

\subsection{Stability of Pest-Free Periodic Solutions}

First, we present a condition which guarantees the stability for the prey-free periodic solution $\left(0, y^{*}(t)\right)$. 
Theorem 3.1. If $d r \tau+d \lambda((1-\cos (\omega \tau)) / \omega)<p$, then the pest-free periodic solution $\left(0, y^{*}(t)\right)$ is locally asymptotically stable. Moreover, if $d \tau(r+\lambda)\left(r^{2}+e K^{2}(r+\lambda)^{2}\right) / r^{2}<p$, then the solution $\left(0, y^{*}(t)\right)$ is globally asymptotically stable.

Proof. The local stability of the periodic solution $\left(0, y^{*}(t)\right)$ can be determined by considering the behavior of small amplitude perturbations of the solution. Define $x(t)=u(t), y(t)=$ $y^{*}(t)+v(t)$. Then they may be written as

$$
\left(\begin{array}{l}
u(t) \\
v(t)
\end{array}\right)=\Phi(t)\left(\begin{array}{l}
u(0) \\
v(0)
\end{array}\right), \quad 0 \leq t \leq \tau,
$$

where $\Phi(t)$ satisfies

$$
\frac{d \Phi}{d t}=\left(\begin{array}{cc}
r+\lambda \sin (\omega t)-y^{*}(t) & 0 \\
q y^{*}(t) & -d
\end{array}\right) \Phi(t)
$$

and $\Phi(0)=I$, where $I$ is the identity matrix. The linearization of the third and fourth equation of system (1.5) becomes

$$
\left(\begin{array}{l}
u\left(n \tau^{+}\right) \\
v\left(n \tau^{+}\right)
\end{array}\right)=\left(\begin{array}{ll}
1 & 0 \\
0 & 1
\end{array}\right)\left(\begin{array}{l}
u(n \tau) \\
v(n \tau)
\end{array}\right)
$$

Note that all eigenvalues of $S=\left(\begin{array}{ll}1 & 0 \\ 0 & 1\end{array}\right) \Phi(\tau)$ are $0<\mu_{1}=\exp (-d \tau)<1$ and $0<\mu_{2}=\exp \left(\int_{0}^{\tau} r+\right.$ $\left.\lambda \sin (\omega t)-y^{*}(t) d t\right)$. Since $\int_{0}^{\tau} y^{*}(t) d t=p / d$, the condition $\mu_{2}<1$ is equivalent to the equation

$$
r \tau+\lambda\left(\frac{1-\cos (\omega \tau)}{\omega}\right)-\frac{p}{d}<0
$$

According to Floquet theory $[17,18],\left(0, y^{*}(t)\right)$ is locally stable.

Next, to prove the global stability of $\left(0, y^{*}(t)\right)$, let $(x(t), y(t))$ be any solution of system (1.5). Simple calculations yield that $(1-\cos (\omega \tau)) / \omega \leq \tau$. So, under the condition $d \tau(r+$ $\lambda)\left(r^{2}+e K^{2}(r+\lambda)^{2}\right) / r^{2}<p$, we know that $\left(0, y^{*}(t)\right)$ maintains the local stability and can take a sufficiently small number $\epsilon>0$ satisfying

$$
\zeta=\exp \left(\left(r+\lambda+\epsilon_{1}\right) \tau-\frac{p r^{2}}{d\left(r^{2}+e K^{2}\left(r+\lambda+r^{2} \epsilon_{1}\right)^{2}\right)}\right)<1
$$


It follows from the first equation in (1.5) that $x^{\prime}(t) \leq x(t)(r+\lambda-(r / K) x(t))$ for $t \neq n \tau$. Then, from Lemma 2.3, we have $x(t) \leq v(t)$, where $v(t)$ is a solution of the following impulsive differential equation:

$$
\begin{gathered}
v^{\prime}(t)=v(t)\left(r+\mathcal{l}-\frac{r}{K} v(t)\right), \quad t \neq n \tau, \\
\Delta v(t)=0, \quad t=n \tau \\
v\left(0^{+}\right)=x\left(0^{+}\right) .
\end{gathered}
$$

Since $v(t) \rightarrow K(r+\lambda) / r$ as $t \rightarrow \infty, x(t) \leq K(r+\lambda) / r+\epsilon_{1}$ for any $\epsilon_{1}>0$ with $t$ large enough. For simplicity we may assume that $x(t) \leq K(r+\lambda) / r+\epsilon_{1}$ for all $t>0$. Since $y^{\prime}(t) \geq-d y(t)$ for $t \neq n \tau$, we can obtain from Lemmas 2.3 and 2.6 that

$$
y(t)>y^{*}(t)-\epsilon_{1}
$$

for $t$ sufficiently large. Without loss of generality, we may assume that (3.7) holds for all $t \geq 0$. From (1.5) and (3.7) we obtain

$$
\begin{gathered}
x^{\prime}(t) \leq x(t)\left(r+\lambda-\frac{\left(y^{*}(t)-\epsilon_{1}\right)}{1+e\left(K(r+\lambda) / r+\epsilon_{1}\right)^{2}}\right), \quad t \neq n \tau, \\
\Delta x(t)=0, \quad t=n \tau .
\end{gathered}
$$

Integrating (3.8) on $((n-1) \tau, n \tau]$, we get $x(n \tau) \leq x((n-1) \tau) \zeta^{n}$ and thus $x(n \tau) \leq x\left(0^{+}\right) \zeta^{n}$ which implies that $x(n \tau) \rightarrow 0$ as $n \rightarrow \infty$. Further, for $t \in((n-1) \tau, n \tau]$, we obtain

$$
\begin{aligned}
x(t) & \leq x((n-1) \tau+) \exp \left(\int_{(n-1) \tau}^{t} r+\lambda-\frac{\left(y^{*}(t)-\epsilon_{1}\right)}{1+e\left(K(r+\lambda) / r+\epsilon_{1}\right)^{2}} d t\right) \\
& \leq x((n-1) \tau) \exp \left(\left(r+\lambda+\epsilon_{1}\right) \tau\right)
\end{aligned}
$$

which implies that $x(t) \rightarrow 0$ as $t \rightarrow \infty$. Now, take a sufficiently small numbers $\epsilon_{2}>0$ satisfying $c \epsilon_{2}<d$ to prove that $y(t) \rightarrow y^{*}(t)$ as $t \rightarrow \infty$. Since $\lim _{t \rightarrow \infty} x(t)=0$, we may assume that $x(t) \leq \epsilon_{2}$ for all $t \geq 0$. It follows from the second equation in (1.5) that, for $t \neq n \tau$,

$$
y^{\prime}(t) \leq y(t)\left(-d+c \epsilon_{2}\right)
$$

Thus, by Lemma 2.3, we induce that $y(t) \leq \tilde{y}^{*}(t)$, where $\tilde{y}^{*}(t)$ is the solution of (2.7) with $d$ changed into $d-c \epsilon_{2}$. Therefore, by taking sufficiently small $\epsilon_{2}$, we obtain from Lemma 2.6 and (3.7) that $y(t)$ tends to $y^{*}(t)$ as $t \rightarrow \infty$.

Remark 3.2. Since $(1-\cos (\omega \tau)) / \omega \leq \tau$, we can obtain a condition without $\omega$ that $\left(0, y^{*}(t)\right)$ is locally stable. In other words, if the condition $(r+\lambda) d \tau<p$ holds, then the prey-free periodic solution $\left(0, y^{*}(t)\right)$ is locally stable. Now, in order to substantiate our theoretical results, let 


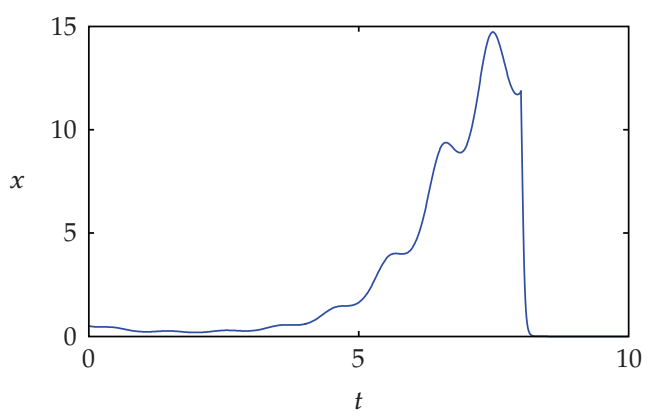

(a)

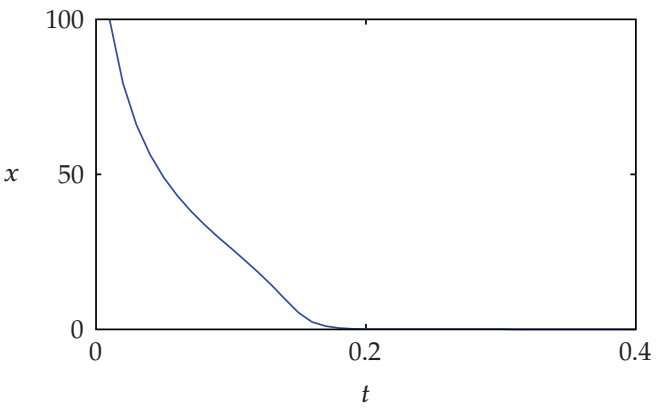

(c)

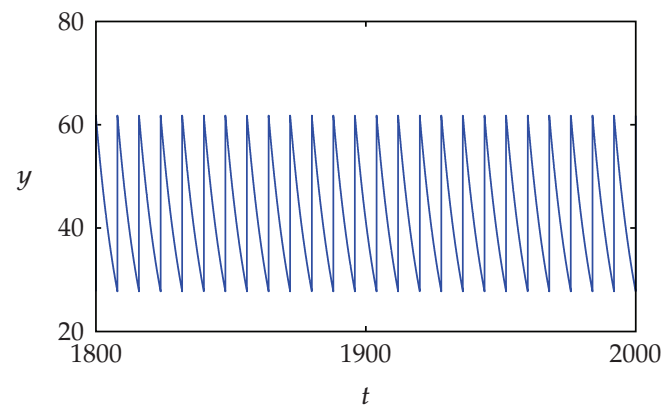

(b)

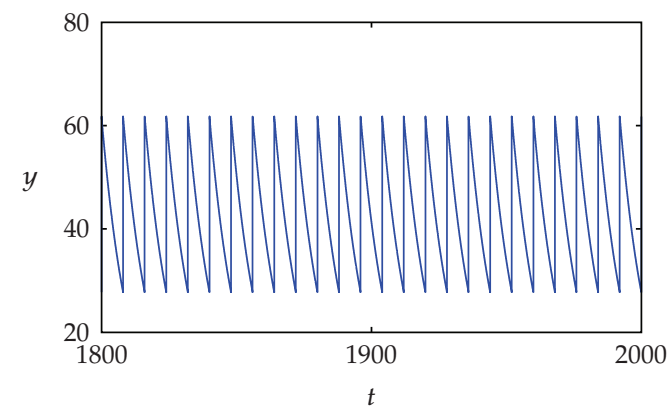

(d)

Figure 1: (a)-(b) Time series of $x$ and $y$ when $p=34$ and $\left(x_{0}, y_{0}\right)=(0.5,7)$ and (c)-(d) time series of $x$ and $y$ when $p=34$ and $\left(x_{0}, y_{0}\right)=(100,100)$.

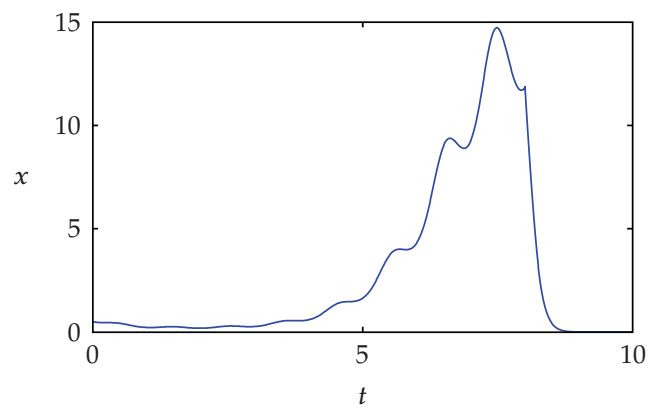

(a)

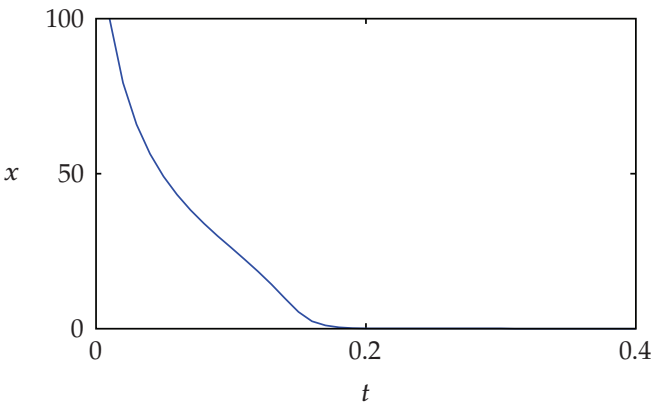

(c)

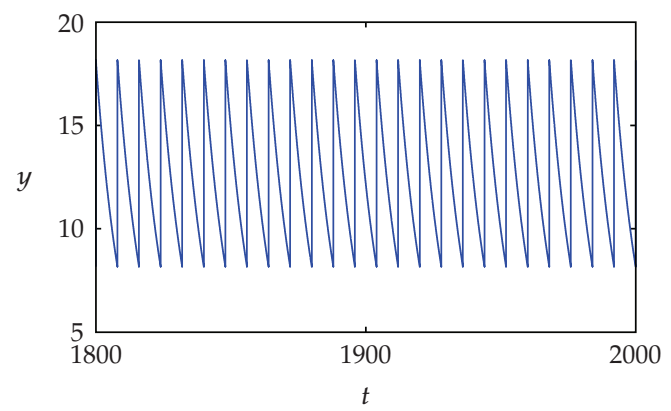

(b)

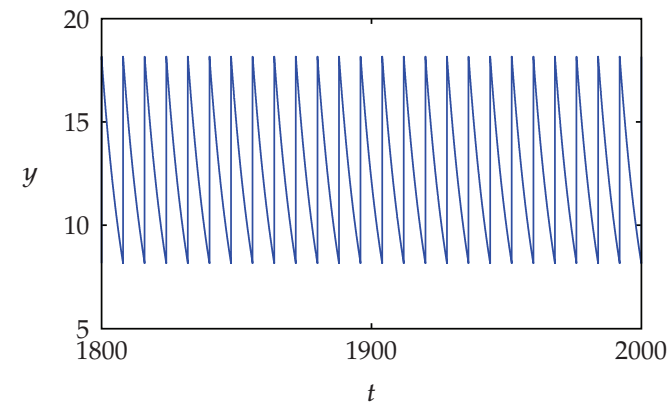

(d)

Figure 2: (a)-(b) Time series of $x$ and $y$ when $p=10$ and $\left(x_{0}, y_{0}\right)=(0.5,7)$ and (c)-(d) time series of $x$ and $y$ when $p=10$ and $\left(x_{0}, y_{0}\right)=(100,100)$. 
$r=6, K=19, d=0.1, c=0.025, e=0.01, \omega=2 \pi, \lambda=1$, and $\tau=8$. It follows from Theorem 3.1 that system (1.5) is locally stable if the condition $p>4.8$ holds and is globally stable if the condition $p>33.11$ holds (see Figure 1). Further, Figure 2 indicates that system (1.5) may be globally stable even if $4.8<p<33.11$.

\subsection{Permanence}

Before discussing permanence we set up its definition.

Definition 3.3. System (1.5) is permanent if there exist $M \geq m>0$ such that any solution $(x(t), y(t))$ of system (1.5) with $x_{0}>0, y_{0}>0$ satisfies

$$
m \leq \lim _{t \rightarrow \infty} \inf x(t) \leq \lim _{t \rightarrow \infty} \sup x(t) \leq M, \quad m \leq \lim _{t \rightarrow \infty} \inf y(t) \leq \lim _{t \rightarrow \infty} \sup y(t) \leq M
$$

Theorem 3.4. System (1.5) is permanent if $(r-\lambda) d \tau>p$.

Proof. Let $(x(t), y(t))$ be a solution of system (1.5) with $x_{0}>0$ and $y_{0}>0$. From Theorem 2.7, we may assume that $x(t) \leq M, y(t) \leq M, t \geq 0$, and $M>r-\lambda$. Let $m_{2}=p \exp (-d \tau) /(1-$ $\exp (-d \tau))-\epsilon_{2}, \epsilon_{2}>0$. From the second and the forth equations of system (1.5), we obtain that $y^{\prime}(t) \geq-d y(t)$ when $t \neq n \tau$ and $y\left(n \tau^{+}\right)=y(n \tau)+p$. So, it follows from Lemma 2.6 that $y(t) \geq m_{2}$ for all $t$ that are large enough. Now we will find an $m_{1}>0$ such that $x(t) \geq m_{1}$ for all $t$ that are large enough. To do this we will take the following two steps.

Step 1. Since $(r-\lambda) d \tau>p$, we can choose $m_{3}>0, \epsilon_{1}>0$ small enough such that $\delta=c m_{3}<d$ and $\eta=\left(r-\lambda-\epsilon_{1}-(r / K) m_{3}\right) \tau-p /(d-\delta)>0$. Suppose that $x(t)<m_{3}$ for all $t$. Then it follows from (1.5) that $y^{\prime}(t) \leq y(t)(-d+\delta)$. By Lemma 2.3, we have $y(t) \leq u(t)$ and $u(t) \rightarrow u^{*}(t)$ as $t \rightarrow \infty$, where $u(t)$ is a solution of

$$
\begin{gathered}
u^{\prime}(t)=(-d+\delta) u(t), \quad t \neq n \tau, \\
u\left(t^{+}\right)=u(t)+p, \quad t=n \tau, \\
u\left(0^{+}\right)=u_{0}>0,
\end{gathered}
$$

and $u^{*}(t)=p \exp ((-d+\delta)(t-n \tau)) /(1-\exp ((-d+\delta) \tau)), t \in((n-1) \tau, n \tau]$. Then there exists $\tau_{1}>0$ such that, for $t \geq \tau_{1}, y(t) \leq u(t) \leq u^{*}(t)+\epsilon_{1}$ and

$$
\begin{aligned}
x^{\prime}(t) & =x(t)\left(r+\lambda \sin (\omega t)-\frac{r}{K} x(t)-\frac{y(t)}{1+e x^{2}(t)}\right) \\
& \geq x(t)\left(r-\lambda-\frac{r}{K} m_{3}-y(t)\right) \\
& \geq x(t)\left(r-\lambda-\frac{r}{K} m_{3}-\left(u^{*}(t)+\epsilon_{1}\right)\right) .
\end{aligned}
$$


Let $N_{1} \in \mathbb{N}$ and $N_{1} \tau \geq \tau_{1}$. Integrating (3.13) on $((n-1) \tau, n \tau], n \geq N_{1}$, we obtain $x(n \tau) \geq$ $x((n-1) \tau) \exp \left(\int_{(n-1) \tau}^{n \tau} r-\lambda-(r / K) m_{3}-\left(u^{*}(t)+\epsilon_{1}\right) d t\right) \geq x\left((n-1) \tau^{+}\right) \exp (\eta)$. Then $x\left(\left(N_{1}+k\right) \tau\right) \geq$ $x\left(N_{1} \tau^{+}\right) \exp (k \eta) \rightarrow \infty$ as $k \rightarrow \infty$ which is a contradiction to Theorem 2.7. Hence there exists a $t_{1}>0$ such that $x\left(t_{1}\right) \geq m_{3}$.

Step 2. If $x(t) \geq m_{3}$ for all $t>t_{1}$, then the proof is complete. If not, let $t^{*}=\inf \{t: t>$ $\left.t_{1}, x(t)<m_{3}\right\}$. Then $x(t) \geq m_{3}$ for $t \in\left(t_{1}, t^{*}\right]$ and, by the continuity of $x(t)$, we have $x\left(t^{*}\right)=m_{3}$. Suppose that $t^{*} \in\left[n_{1} \tau,\left(n_{1}+1\right) \tau\right)$ for some $n_{1} \in \mathbb{N}$. Since $M>r-\lambda$ and $m_{3}>0$ is small enough, we may assume that $\eta_{1} \equiv r-\lambda-(r / K) m_{3}-M<0$. Select $n_{2}, n_{3} \in \mathbb{N}$ such that $n_{2} \tau>\log \left(\epsilon_{1} /(M+p)\right) /(-d+\delta)$ and $\exp \left(\left(n_{2}+1\right) \eta_{1} \tau\right) \exp \left(n_{3} \eta\right)>1$. Let $T=n_{2} \tau+n_{3} \tau$. Then there are two possible cases for $t \in\left(t^{*},\left(n_{1}+1\right) \tau\right]$.

Case 1. For all $t \in\left(t^{*},\left(n_{1}+1\right) \tau\right], x(t)<m_{3}$.

In this case we will show that there exists $t^{\prime} \in\left[\left(n_{1}+1\right) \tau,\left(n_{1}+1\right) \tau+T\right]$ such that $x\left(t^{\prime}\right) \geq m_{3}$. If not, we have $x(t)<m_{3}$ for all $t \in\left[\left(n_{1}+1\right) \tau,\left(n_{1}+1+n_{2}+n_{3}\right) \tau\right]$. Then $x(t)<m_{3}$ for all $t \in\left(t^{*},\left(n_{1}+1+n_{2}+n_{3}\right) \tau\right]$. It follows from (1.5) that $y^{\prime}(t) \leq\left(-d+c m_{3}\right) y(t)=(-d+\delta) y(t)$ for $t \neq n \tau$. By (3.12) with $u\left(\left(n_{1}+1\right) \tau^{+}\right)=y\left(\left(n_{1}+1\right) \tau^{+}\right)$, we have

$$
u(t)=\left(u\left(\left(n_{1}+1\right) \tau^{+}\right)-\frac{p}{1-\exp (-d+\delta)}\right) \exp \left((-d+\delta)\left(t-\left(n_{1}+1\right) \tau\right)\right)+u^{*}(t)
$$

for $t \in(n \tau,(n+1) \tau], n_{1}+1 \leq n \leq n_{1}+n_{2}+n_{3}$. So we get $\left|u(t)-u^{*}(t)\right| \leq(M+p) \exp \left((-d+\delta) n_{2} \tau\right)<$ $\epsilon_{1}$. Thus, from Lemma 2.3, we obtain $y(t) \leq u(t) \leq u^{*}(t)+\epsilon_{1}$ for $t \in\left[\left(n_{1}+1+n_{2}\right) \tau,\left(n_{1}+1+n_{2}+\right.\right.$ $\left.\left.n_{3}\right) \tau\right]$. In the same manner as (3.13), it follows that $x^{\prime}(t) \geq x(t)\left(r-\mathcal{\lambda}-(r / K) m_{3}-\left(u^{*}+\epsilon_{1}\right)\right)$ for $t \in\left[\left(n_{1}+1+n_{2}\right) \tau,\left(n_{1}+1+n_{2}+n_{3}\right) \tau\right]$. As in Step 1, we have

$$
x\left(\left(n_{1}+1+n_{2}+n_{3}\right) \tau\right) \geq x\left(\left(n_{1}+1+n_{2}\right) \tau\right) \exp \left(n_{3} \eta\right) .
$$

Since $y(t) \leq M$, we obtain $x^{\prime}(t)=x(t)\left(r-\lambda \sin (\omega t)-(r / K) x(t)-y(t) /\left(1+e x^{2}(t)\right)\right) \geq x(t)(r-$ $\left.\lambda-(r / K) m_{3}-M\right)=\eta_{1} x(t)$ for all $t \in\left(t^{*},\left(n_{1}+1+n_{2}\right) \tau\right]$. Integrating this on $\left[t^{*},\left(n_{1}+1+n_{2}\right) \tau\right]$ we obtain

$$
\begin{aligned}
x\left(\left(n_{1}+1+n_{2}\right) \tau\right) & \geq x\left(t^{*}\right) \exp \left(\eta_{1}\left(n_{1}+1+n_{2}\right) \tau-t^{*}\right) \\
& \geq m_{3} \exp \left(\eta_{1}\left(n_{2}+1\right) \tau\right) .
\end{aligned}
$$

Thus $x\left(\left(n_{1}+1+n_{2}+n_{3}\right) \tau\right) \geq m_{3} \exp \left(\eta_{1}\left(n_{2}+1\right) \tau\right) \exp \left(n_{3} \eta\right)>m_{3}$ which is a contradiction. Now, let $\bar{t}=\inf \left\{t: t>t^{*}, x(t)>m_{3}\right\}$. Then $x(t) \leq m_{3}$ for $t^{*} \leq t<\bar{t}$ and $x(\bar{t})=m_{3}$. So, we have, for $t \in\left[t^{*}, \bar{t}\right), x^{\prime}(t) \geq x(t)\left(r-\lambda-(r / K) m_{3}-M\right)$. By the integration of it on $\left[t^{*}, t\right)\left(t^{*} \leq t \leq \bar{t}\right)$, we can get that $x(t) \geq x\left(t^{*}\right) \exp \left(\eta_{1}\left(t-t^{*}\right)\right) \geq m_{3} \exp \left(\eta_{1}\left(1+n_{2}+n_{3}\right) \tau\right) \equiv m_{1}$. For $t>\bar{t}$, the same arguments can be continued since $x(\bar{t}) \geq m_{3}$.

Case 2. There exists a $t^{\prime} \in\left(t^{*},\left(n_{1}+1\right) \tau\right]$ such that $x\left(t^{\prime}\right) \geq m_{3}$.

Let $\underline{t}=\inf \left\{t: t>t^{*}, x(t)>m_{3}\right\}$. Then $x(t) \leq m_{3}$ for $t \in\left[t^{*}, \underline{t}\right)$ and $x(\underline{t})=m_{3}$. For $t \in\left[t^{*}, \underline{t}\right), x^{\prime}(t) \geq x(t)\left(r-\lambda-(r / K) m_{3}-M\right)$. Integrating this on $\left[t^{*}, t\right)\left(t^{*} \leq t \leq \underline{t}\right)$, we can get that $x(t) \geq x\left(t^{*}\right) \exp \left(\eta_{1}\left(t-t^{*}\right)\right) \geq m_{3} \exp \left(\eta_{1} \tau\right) \geq m_{1}$. For $t>t$, the same arguments can be continued since $x(\underline{t}) \geq m_{3}$. 


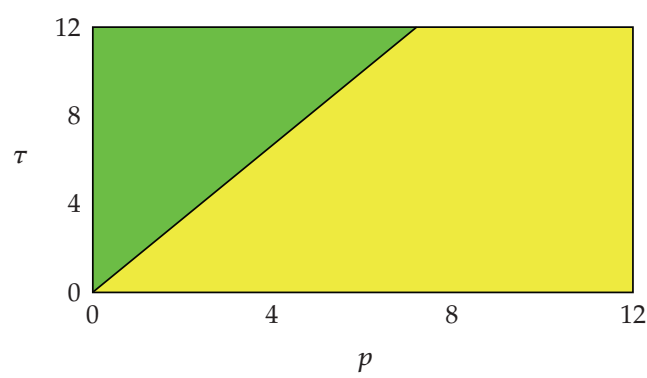

(a)
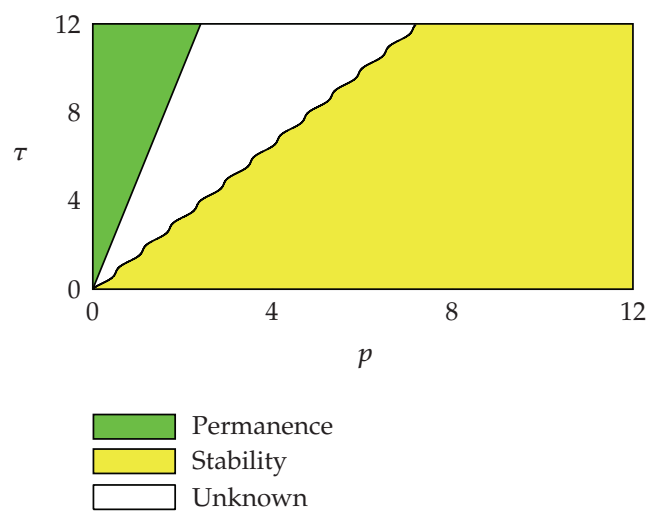

(c)

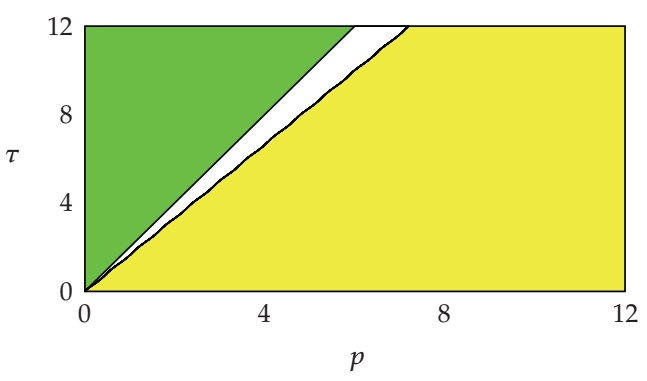

(b)

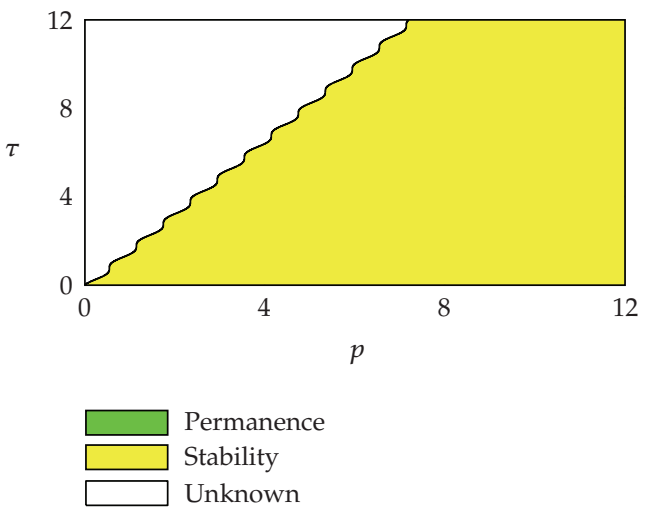

(d)

Figure 3: Regions induced from Theorems 3.1 and 3.4. (a) $\lambda=0$, (b) $\lambda=1$, (c) $\lambda=4$, (d) $\lambda=6$.

So we know that $x(t) \geq m_{1}$ for $t_{1} \leq t \leq t_{2}=\min \{\bar{t}, \underline{t}\}$ since $m_{3} \geq m_{1}$. Moreover, the same arguments as Step 2 can be continued since $x\left(t_{2}\right) \geq m_{3}$. Thus we obtain $x(t) \geq m_{1}$ for $t \geq t_{1}$. Therefore, we complete the proof.

Remark 3.5. We illustrate Figures 5 and 9 as numerical examples of Theorem 3.4. Now assume that $\sin (\omega \tau) \geq 0$ to improve slightly the result of Theorem 3.4. Then, by (1.5), we obtain that

$$
\begin{aligned}
x^{\prime}(t) & =x(t)\left(r+\lambda \sin (\omega t)-\frac{r}{K} x(t)-\frac{y(t)}{1+e x^{2}(t)}\right) \\
& \geq x(t)\left(r+\lambda \sin (\omega t)-\frac{r}{K} m_{3}-\left(u^{*}(t)+\epsilon_{1}\right)\right) .
\end{aligned}
$$

Note that $\int_{(n-1) \tau}^{n \tau} \sin (\omega t) d t=-(1 / \omega)(\cos (n \omega \tau)-\cos ((n-1) \omega \tau)) \geq-(2 / \omega) \sin (\omega \tau / 2)$ since $\sin (\omega \tau) \geq 0$. Thus, using these facts and the method used to prove Theorem 3.4, we get the following Corollary.

Corollary 3.6. Suppose that $\sin (\omega \tau) \geq 0$. Then system (1.5) is permanent if $d r \tau-$ $2 d \lambda \sin (\omega \tau / 2) / \omega>p$. 


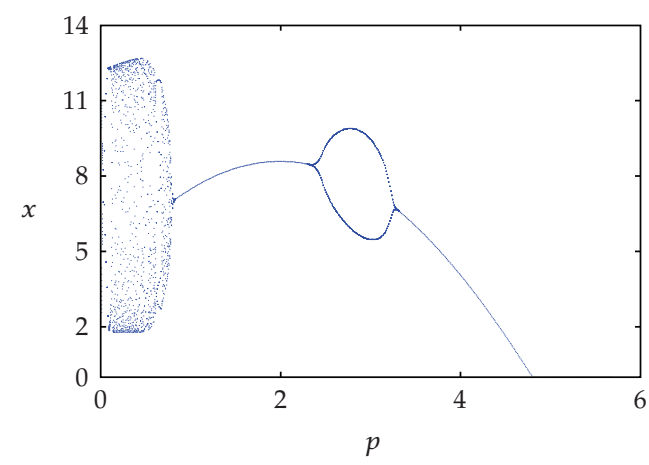

(a)

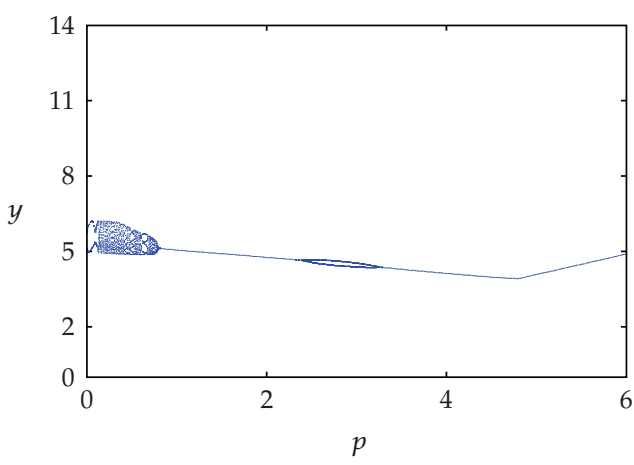

(b)

Figure 4: Bifurcation diagrams of system (1.5) with $\tau=8$ and $\lambda=0$. (a) $x$ is plotted for $p$; (b) $y$ is plotted for $p$.

\section{Numerical Analysis on Seasonal Effects and Impulsive Perturbations}

In this section, we will investigate the influence of periodic forcing and impulsive perturbations. We fix the parameters and an initial condition as follows:

$$
\begin{aligned}
& r=6, \quad K=19, \quad d=0.1, \quad c=0.025, \\
& e=0.01, \quad \omega=2 \pi, \quad x_{0}=0.5, \quad y_{0}=7 .
\end{aligned}
$$

From [5], we know that system (1.3) has an unstable positive equilibrium and a unique stable limit cycle. Zhang et al. [22] have already studied the abundant dynamical behaviors of system (1.5) under the same setting of parameters. Here, we focus on the permanence condition and the control strategy for the population densities of the prey and the predator. Using Theorems 3.1 and 3.4, we display the permanence and stability regions for fixed parameter $\lambda$ shown in Figure 3. First, we assume $\lambda=0$. It follows from Theorems 3.1 and 3.4 that the equation $\tau=p / r d=p / 0.6$ is the border line (see Figure 3(a)), which completely separates the permanence and stability regions. If we consider the prey as a pest and the predator as an enemy of the prey, we should release the predator frequently and abundantly in the aim of exterminating the pest. Or if we regard the prey as a rare variety to be protected, the impulsive perturbations of the predator should occur at rare intervals and their quantity should be very small. Figure 3(a) explains well such situation. For instance, let $\tau=8$ and $\lambda=0$; it follows from Theorems 3.1 and 3.4 that if $p<p_{1}(=4.8)$ the prey and the predator coexist, but the prey is extinct after $p>p_{1}$ (see Figure 4 ).

To study the seasonal effects on the prey, we suppose $\lambda \neq 0$. In this case, the area of unknown region increases according to the value of $\lambda$ (see Figure 3). Let $p$ and $\tau$ be in the unknown region. Then we know from Figures 6 and 7 that two species coexist if $p<p_{2}(\approx 4.8)$, otherwise the prey is extinct. One of interesting things in Figures 4, 6, and 7 is that the fluctuations of the density of the predator are smaller than those of the prey for small $p$, even though there is the influx of the predator periodically. However, the fluctuations of the prey diminishe according to $\lambda$. These facts suggest that releasing the predator on purpose to increase its density may have no effect. On the other hand, abundant releasing and frequent loosing of the predator can cause destroying the prey and eventually the ecosystem. As 


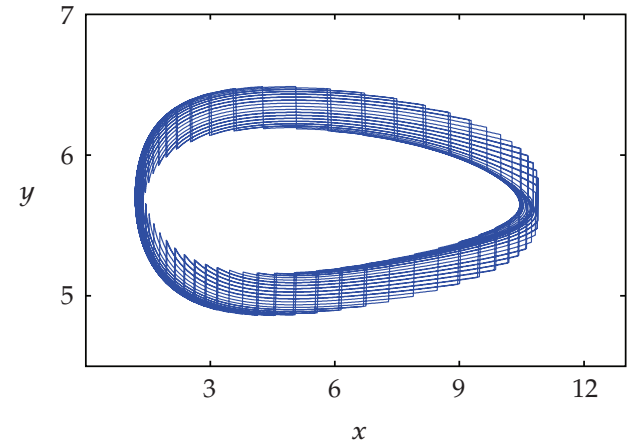

(a)

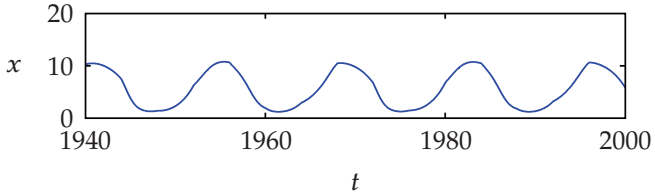

(b)

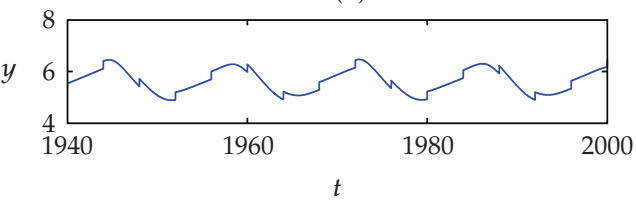

(c)

Figure 5: (a) Phase portrait of system (1.5) with $\tau=4$ and $\lambda=0$ and $p=0.3$, (b) and (c) Time series of $x$ and $y$.

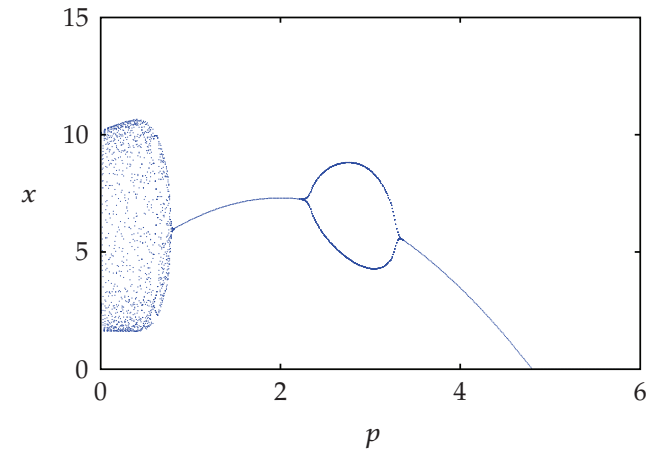

(a)

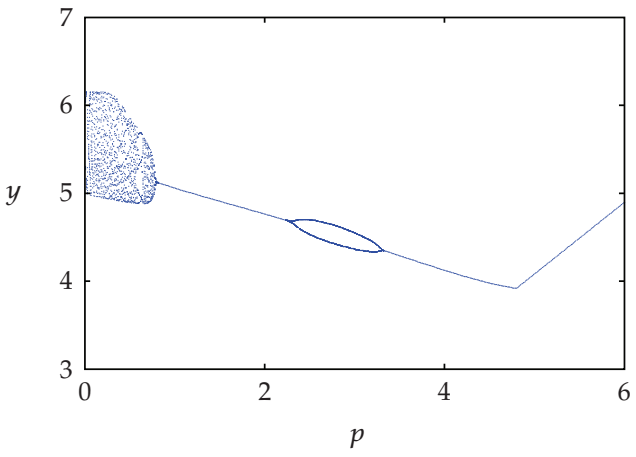

(b)

Figure 6: Bifurcation diagrams of system (1.5) with $\tau=8$ and $\lambda=1$. (a) $x$ is plotted for $p$, (b) $y$ is plotted for $p$.

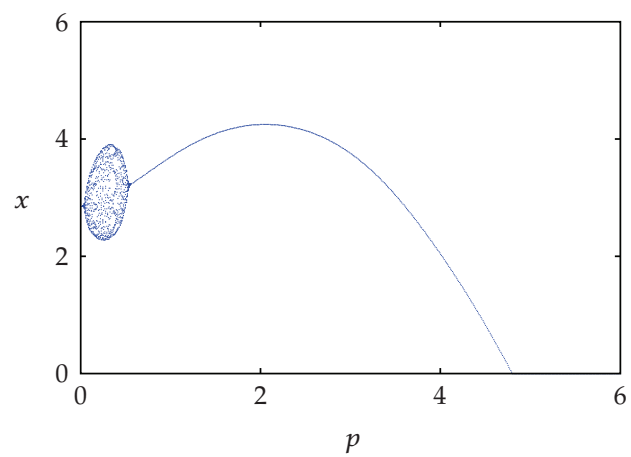

(a)

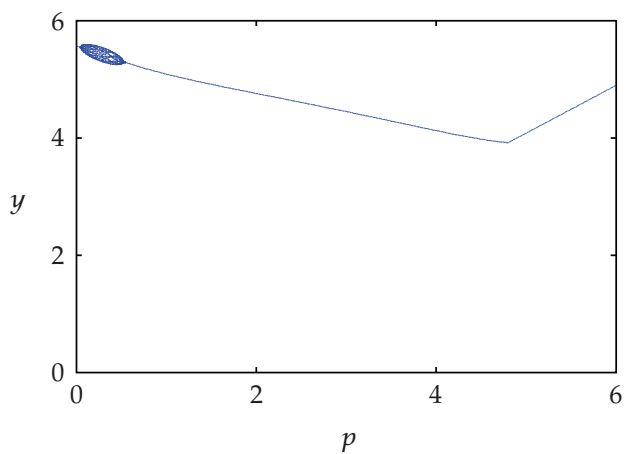

(b)

Figure 7: Bifurcation diagrams of system (1.5) with $\tau=8$ and $\lambda=4$. (a) $x$ is plotted for $p$, (b) $y$ is plotted for $p$. 


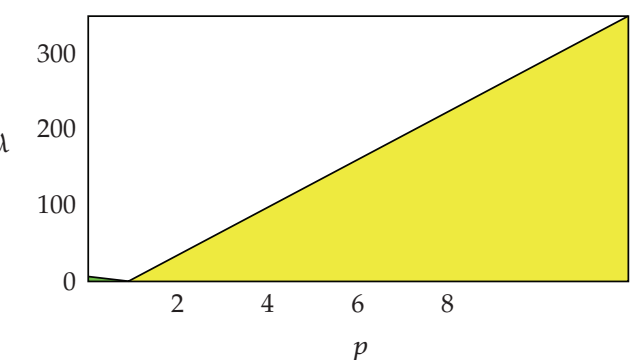

(a)

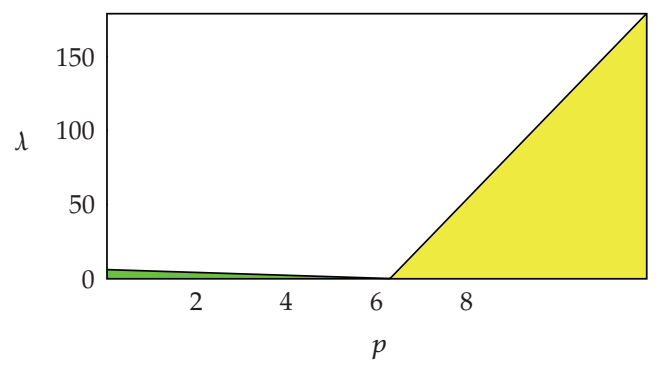

Permanence

Stability

Unknown

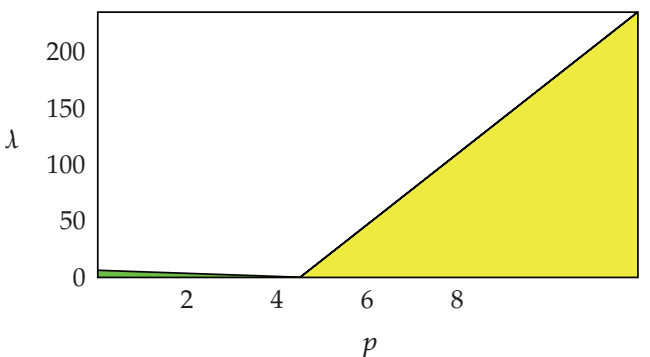

(b)

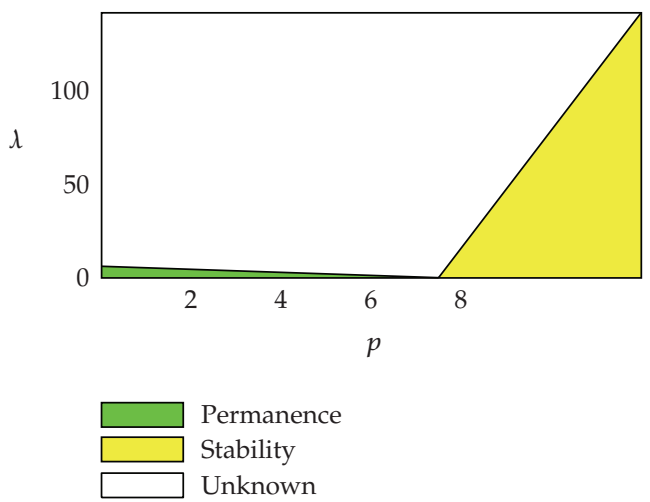

(d)

Figure 8: Regions induced from Theorems 3.1 and 3.4. (a) $\tau=1.5$; (b) $\tau=8.5$; (c) $\tau=10.5$; (d) $\tau=12.5$.

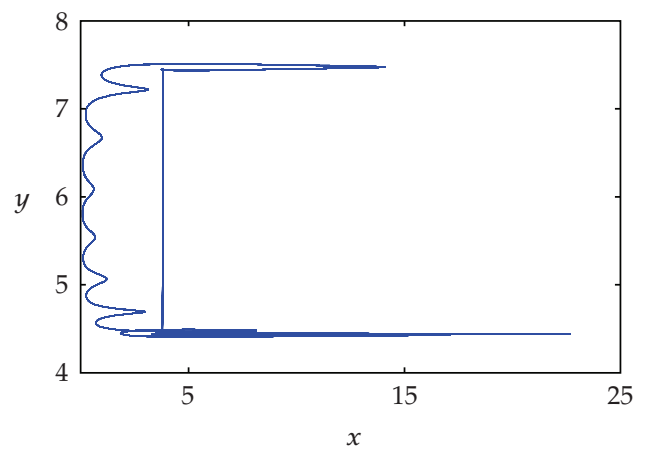

(a)

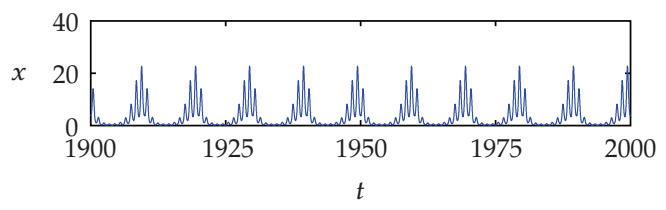

(b)

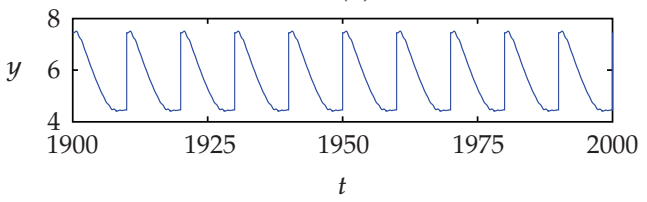

(c)

Figure 9: (a) Phase portrait of system (1.5) with $\lambda=2, \tau=10$, and $p=3$; (b), (c) time series of $x$ and $y$.

shown in Figure 8, we figure out that the longer the period $\tau$ is, the larger the permanence region is and the smaller the stability region is if $\tau$ is not a positive integer.

It means that we should release the predator within a short period to eradicate the prey. On the contrary, impulsive perturbations of the predator should be occurred at long-time intervals for the coexistence of the prey and the predator. If we choose $(\lambda, \tau, p)=(2,10,3)$, we can see the coexistence of the prey and the predator as shown in Figure 9. 


\section{Conclusion}

In this paper, we have investigated the effects of periodic forcing in the intrinsic growth rate of the prey and impulsive perturbations of the predator, on the Monod-Haldane functional response predator-prey model. We have shown that there exists an asymptotically stable prey-free periodic solution if the impulsive period is less than a critical value and have also found parameter regions where system (1.5) is permanent. In addition, we have discussed biological roles of parameters $p, \lambda$, and $\tau$ in Section 4 . Although Theorems 3.1 and 3.4 cannot completely classify the dynamical properties of system (1.5), numerical results supply the border lines which separate two areas having different dynamical properties.

\section{Acknowledgments}

The authors would like to thank the referee for carefully reading of the manuscript and suggesting improvements. This work was supported by the Korea Science and Engineering Foundation (KOSEF) Grant funded by the Korea government (MEST) (no. R01-2008-00020088-0).

\section{References}

[1] C. S. Holling, "The functional response of predator to prey density and its role in mimicry and population regulations," Mem. Ent. Sec. Can, vol. 45, pp. 1-60, 1965.

[2] J. F. Andrews, "A mathematical model for the continuous culture of macroorganisms untilizing inhibitory substrates," Biotechnology and Bioengineering, vol. 10, pp. 707-723, 1968.

[3] W. Sokol and J. A. Howell, "Kineties of phenol oxidation by ashed cell," Biotechnology and Bioengineering, vol. 23, pp. 2039-2049, 1980.

[4] S.-B. Hsu and T.-W. Huang, "Global stability for a class of predator-prey systems," SIAM Journal on Applied Mathematics, vol. 55, no. 3, pp. 763-783, 1995.

[5] S. Ruan and D. Xiao, "Global analysis in a predator-prey system with nonmonotonic functional response," SIAM Journal on Applied Mathematics, vol. 61, no. 4, pp. 1445-1472, 2001.

[6] E. Sáez and E. González-Olivares, "Dynamics of a predator-prey model," SIAM Journal on Applied Mathematics, vol. 59, no. 5, pp. 1867-1878, 1999.

[7] J. Sugie, R. Kohno, and R. Miyazaki, "On a predator-prey system of Holling type," Proceedings of the American Mathematical Society, vol. 125, no. 7, pp. 2041-2050, 1997.

[8] J. M. Cushing, "Periodic time-dependent predator-prey systems," SIAM Journal on Applied Mathematics, vol. 32, no. 1, pp. 82-95, 1977.

[9] S. Gakkhar and R. K. Naji, "Chaos in seasonally perturbed ratio-dependent prey-predator system," Chaos, Solitons E Fractals, vol. 15, no. 1, pp. 107-118, 2003.

[10] G. C. W. Sabin and D. Summers, "Chaos in a periodically forced predator-prey ecosystem model," Mathematical Biosciences, vol. 113, no. 1, pp. 91-113, 1993.

[11] G. J. Ackland and I. D. Gallagher, "Stabilization of large generalized Lotka-Volterra foodwebs by evolutionary feedback," Physical Review Letters, vol. 93, no. 15, Article ID 158701, 4 pages, 2004.

[12] G. Jiang and Q. Lu, "The dynamics of a prey-predator model with impulsive state feedback control," Discrete and Continuous Dynamical Systems. Series B, vol. 6, no. 6, pp. 1301-1320, 2006.

[13] X. Liu and L. Chen, "Complex dynamics of Holling type II Lotka-Volterra predator-prey system with impulsive perturbations on the predator," Chaos, Solitons E Fractals, vol. 16, no. 2, pp. 311-320, 2003.

[14] B. Liu, Y. Zhang, and L. Chen, "Dynamic complexities in a Lotka-Volterra predator-prey model concerning impulsive control strategy," International Journal of Bifurcation and Chaos in Applied Sciences and Engineering, vol. 15, no. 2, pp. 517-531, 2005.

[15] K. Negi and S. Gakkhar, "Dynamics in a Beddington-DeAngelis prey-predator system with impulsive harvesting," Ecological Modelling, vol. 206, no. 3-4, pp. 421-430, 2007.

[16] X. Song and Y. Li, "Dynamic behaviors of the periodic predator-prey model with modified LeslieGower Holling-type II schemes and impulsive effect," Nonlinear Analysis: Real World Applications, vol. 9, no. 1, pp. 64-79, 2008. 
[17] D. D. Bainov and P. S. Simeonov, Impulsive Differential Equations: Periodic Solutions and Applications, vol. 66 of Pitman Monographs and Surveys in Pure and Applied Mathematics, Longman Science \& Technical, Harlo, UK, 1993.

[18] V. Lakshmikantham, D. D. Baĭnov, and P. S. Simeonov, Theory of Impulsive Differential Equations, vol. 6 of Series in Modern Applied Mathematics, World Scientific, Teaneck, NJ, USA, 1989.

[19] H. Baek, "Dynamic complexites of a three-species Beddington-DeAngelis system with impulsive control strategy," Acta Applicandae Mathematicae, pp. 1-16, 2008.

[20] W. Wang, H. Wang, and Z. Li, "The dynamic complexity of a three-species Beddington-type food chain with impulsive control strategy," Chaos, Solitons \& Fractals, vol. 32, no. 5, pp. 1772-1785, 2007.

[21] Z. Xiang and X. Song, "The dynamical behaviors of a food chain model with impulsive effect and Ivlev functional response," Chaos, Solitons E Fractals, vol. 39, no. 5, pp. 2282-2293, 2009.

[22] S. Zhang, D. Tan, and L. Chen, "Chaos in periodically forced Holling type IV predator-prey system with impulsive perturbations," Chaos, Solitons E Fractals, vol. 27, no. 4, pp. 980-990, 2006.

[23] S. Zhang and L. Chen, "A study of predator-prey models with the Beddington-DeAnglis functional response and impulsive effect," Chaos, Solitons E Fractals, vol. 27, no. 1, pp. 237-248, 2006. 


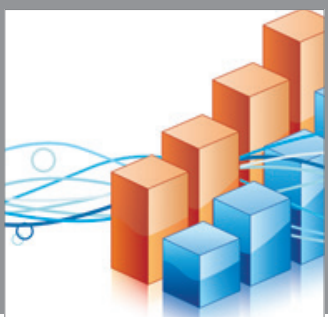

Advances in

Operations Research

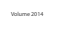

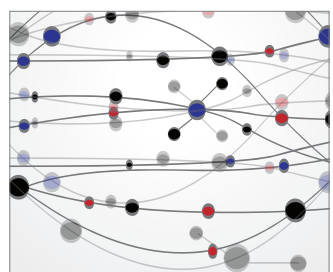

\section{The Scientific} World Journal
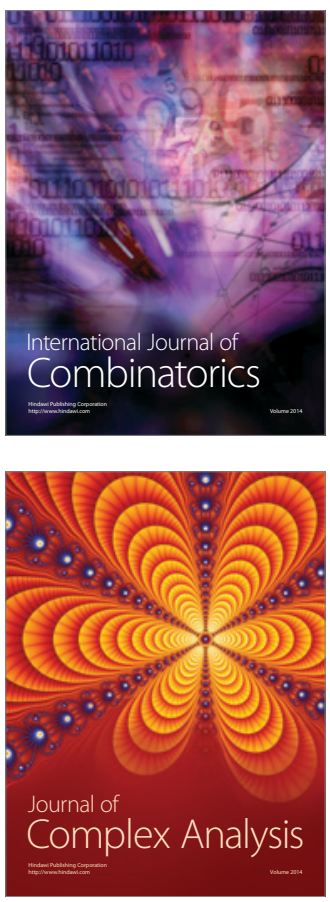

International Journal of

Mathematics and

Mathematical

Sciences
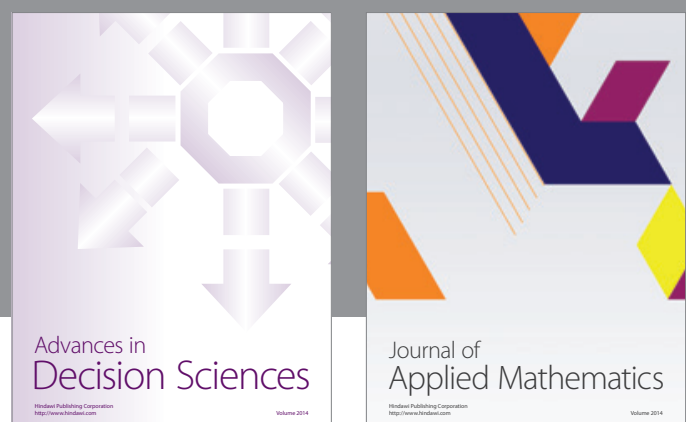

Journal of

Applied Mathematics
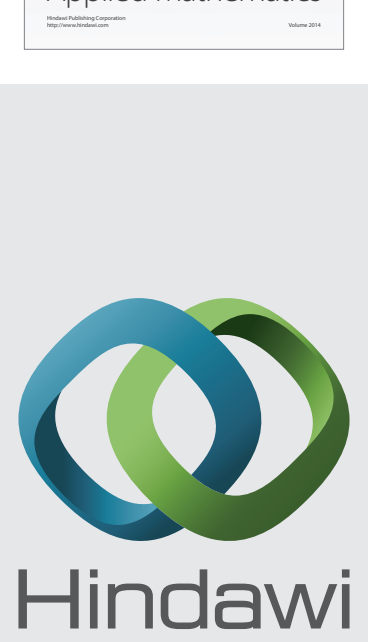

Submit your manuscripts at http://www.hindawi.com
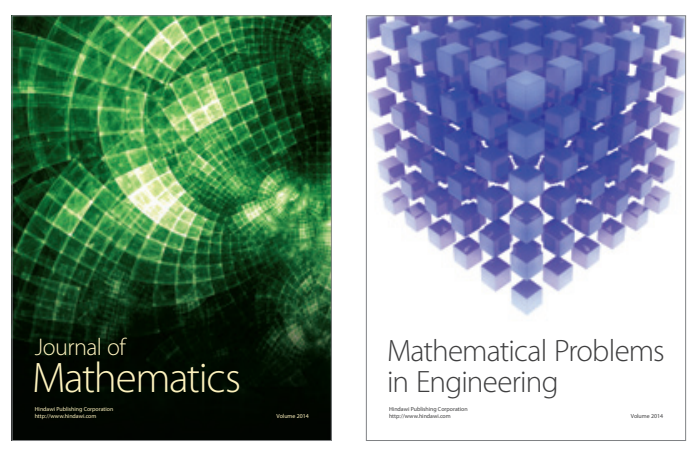

Mathematical Problems in Engineering
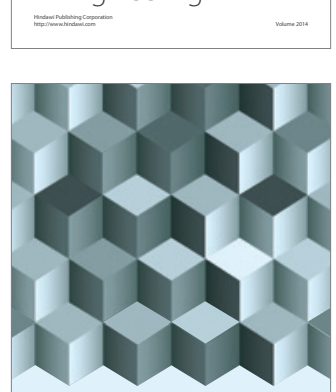

Journal of

Function Spaces
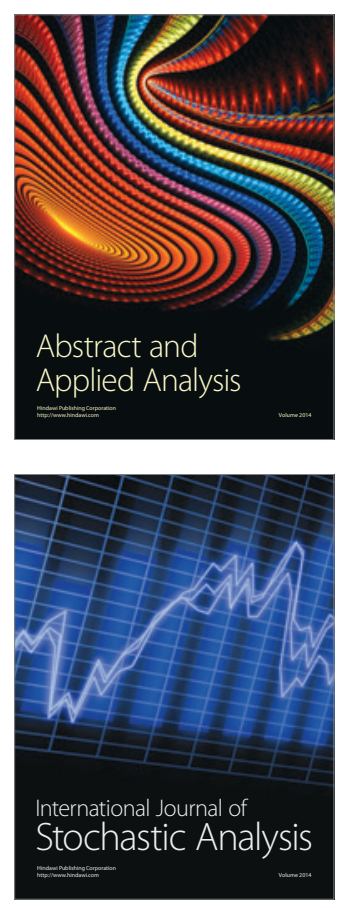

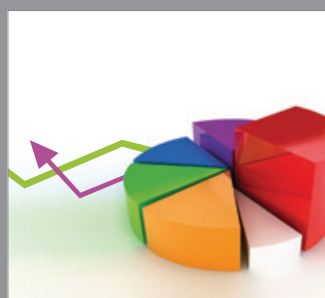

ournal of

Probability and Statistics

Promensencen
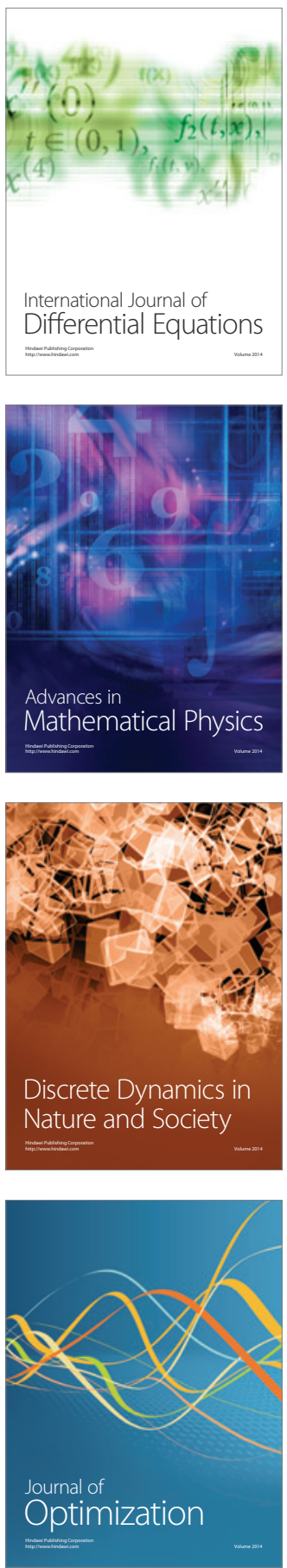